\title{
Adaptive combining of coherently detected optical PPM signals in the presence of atmospheric turbulence via focal plane arrays
}

Michela Munoz Fernandez, Victor A. Vilnrotter

Michela Munoz Fernandez, Victor A. Vilnrotter, "Adaptive combining of coherently detected optical PPM signals in the presence of atmospheric turbulence via focal plane arrays," Proc. SPIE 5572, Optics in Atmospheric Propagation and Adaptive Systems VII, (11 November 2004); doi: $10.1117 / 12.565301$

SPIE. Event: Remote Sensing, 2004, Maspalomas, Canary Islands, Spain 


\title{
Adaptive combining of coherently detected optical PPM signals in the presence of atmospheric turbulence via focal plane arrays
}

\author{
Michela Muñoz Fernández*a,b, Victor A. Vilnrotter ${ }^{\mathrm{b}}$ \\ ${ }^{a}$ California Institute of Technology, 1200 E. California Blvd., Pasadena, CA 91125 \\ ${ }^{\mathrm{b}}$ Jet Propulsion Laboratory, California Institute of Technology, 4800 Oak Grove Dr., Pasadena, CA 91109
}

\begin{abstract}
Adaptive combining of experimentally obtained heterodyned pulse position modulated (PPM) signals with pulse-topulse coherence, in the presence of simulated spatial distortions resembling atmospheric turbulence, is demonstrated. The adaptively combined PPM signals are phased up via an LMS algorithm suitably optimized to operate with PPM in the presence of additive shot-noise. A convergence analysis of the algorithm is presented, and results with both computer simulated and experimentally obtained PPM signals are analyzed.
\end{abstract}

Keywords: Coherent optical communications, pulse position modulation, focal plane array.

\section{INTRODUCTION}

The use of a laser beam as a carrier for a satellite-to-ground link enables transmission using very narrow beam divergence angles. Inhomogeneity in the temperature and pressure of the atmosphere leads to variations of the refractive index and the transmission path. Since the index of refraction of air is not uniform, it distorts the electromagnetic wave passing through it. Therefore, a laser beam traversing the atmosphere is constantly being refracted, or bent and as a result scintillation occurs [1]. This turbulence-induced fading impairs free-space optical links in much the same way that flat multipath fading impairs radio-frequency wireless links. These variations of refracted index as well as pointing vibrations can cause fluctuations in the intensity and phase of the received signal leading to an increase in link error probability.

Absorption by water vapor reduces the energy content in the communication beam, and turbulence increases the beam's divergence. The three main atmospheric processes that affect optical wave propagation are absorption, scattering, and refractive-index fluctuations. Index of refraction fluctuations lead to irradiance fluctuations, beam broadening, and loss of spatial coherence of the optical wave at the receiver. In the context of optical communications, this randomization of the optical phase-front often requires the use of larger receiver field of view, thus admitting more unwanted background radiation into the receiver. In the presence of background radiation, performance of direct detection optical receivers often degrades significantly. One way to overcome the effects of background radiation is to use coherent detection, which is generally much less sensitive to background effects than direct detection [2]. In addition, detectors used for coherent detection have higher quantum efficiency than those used for direct detection photon-counting applications. The solution proposed here is to use focal-plane arrays to collect optical signals from different spatial modes of the received signal field simultaneously, and then recombine the signals optimally. Analysis and proof-of-concept demonstration of coherent adaptive array detection with PPM signals will be described in the following sections.

The research described in this publication was carried out at the Jet Propulsion Laboratory, California Institute of Technology, under a contract with the National Aeronautics and Space Administration.

*E-mail:michela@caltech.edu, phone 1818 354-9272; fax 1818393 1717; caltech.edu 
With coherent detection, the local oscillator mixes with the modulated wave at the surface of the photodetector as shown in Fig. 1. [3]

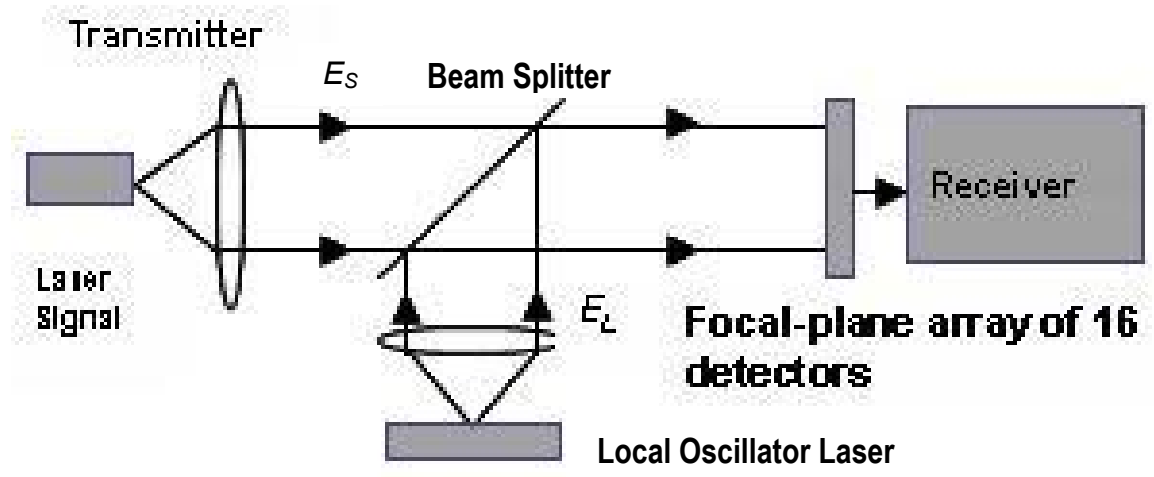

Fig. 1: Configuration of the coherent optical receiver.

The coherent detector converts phase changes in the optical carrier to phase changes in the optical intensity, which are reproduced in the detected current waveform. More detailed analysis was already shown in [4], where Eqs. (1), (2), (3), and (4) were derived. Eqs. (1) and (2) provide the signal-to-noise-ratio (SNR) expression for the homodyne and heterodyne receiver respectively, where $K_{S}$ is the average number of signal photons per pulse.

$$
\begin{aligned}
& S N R(\text { hom })=4 K_{S} . \\
& S N R(\text { het })=2 K_{S} .
\end{aligned}
$$

Equations (3) and (4) show the bit error probabilities for both homodyne and heterodyne detection schemes, where M is the PPM order used,

$$
\begin{aligned}
P_{e} & =\frac{M / 2}{M-1}\left[1-\left\{\int_{-\infty}^{+\infty} \frac{1}{\sqrt{2 \pi}} e^{-\left(w-\sqrt{4 K_{s}}\right)^{2} / 2} d w[1-Q(w)]^{M-1}\right\}\right] \\
& =\frac{M / 2}{M-1}\left[1-\left\{\int_{-\infty}^{+\infty} \frac{1}{\sqrt{2 \pi}} e^{-\left(w-\sqrt{4 K_{s}}\right)^{2} / 2} d w\left[\frac{1}{2}-\frac{1}{2} \operatorname{erf}\left(\frac{w}{\sqrt{2}}\right)\right]^{M-1}\right\}\right] . \\
P_{e} & =\frac{M / 2}{M-1}\left[1-\left\{\int_{-\infty}^{+\infty} \frac{1}{\sqrt{2 \pi}} e^{-\left(w-\sqrt{2 K_{s}}\right)^{2} / 2} d w\left[\frac{1}{2}-\frac{1}{2} \operatorname{erf}\left(\frac{w}{\sqrt{2}}\right)\right]^{M-1}\right\}\right] .
\end{aligned}
$$

Figs. (2) and (3) illustrate the corresponding bit error probabilities obtained with equations (3) and (4) for PPM orders of $2,4,8$ and 16 . 


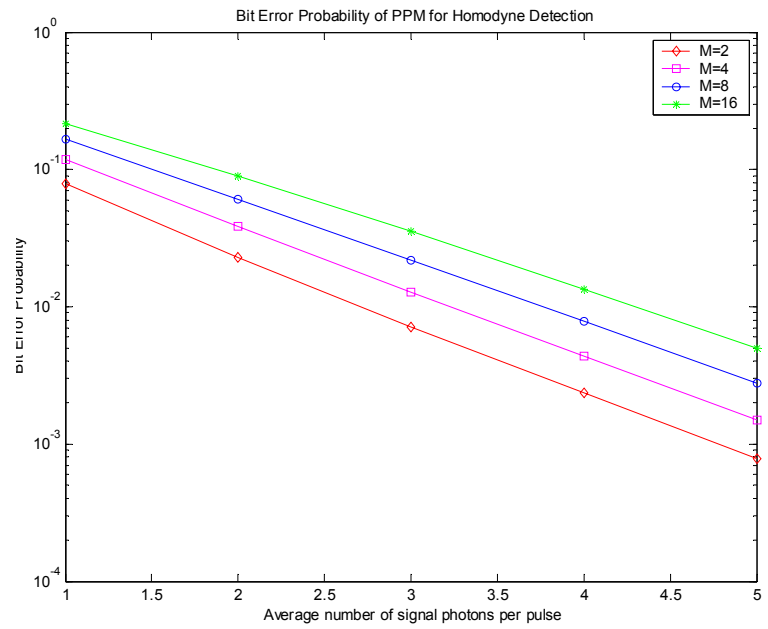

Fig. 2: Bit Error Probability of PPM for homodyne detection.

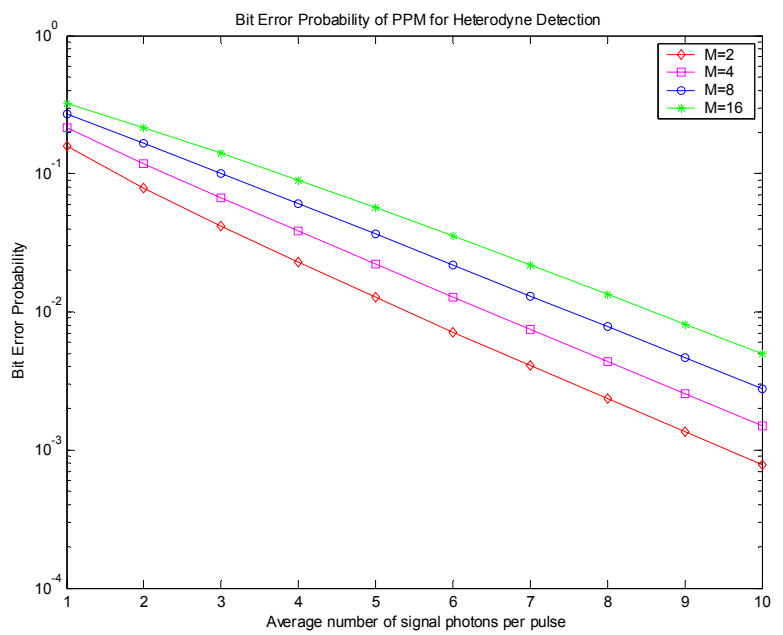

Fig 3.: Bit Error Probability of PPM for heterodyne detection.

\section{EXPERIMENT DESCRIPTION}

The experimental setup of the optical coherent combining experiment consists of two Nd:YAG lasers operating at 1064 nanometers, whose outputs are aligned and combined on the surface of a 4X4 detector array. One of the lasers serves as a local oscillator while the other simulates the received signal. It is a heterodyne detection receiver where the two lasers are operated at slightly different wavelengths, yielding a relatively stable difference-frequency tone of approximately $6 \mathrm{MHz}$ in the detected signal. The difference-frequency tone is generally observed in several array elements simultaneously, but usually with different phases. If the detector element outputs were simply summed, the addition of out-of-phase signal components could result in significant cancellation, yielding a weak signal tone at the output. However, if individual detector elements over which the signal field is essentially coherent are processed separately, then the outputs can be phase-aligned prior to addition, effectively recovering the lost signal power.

Fig. 4 is a photograph of the optical setup at the Jet Propulsion Laboratory where the experiments described in this article have taken place.

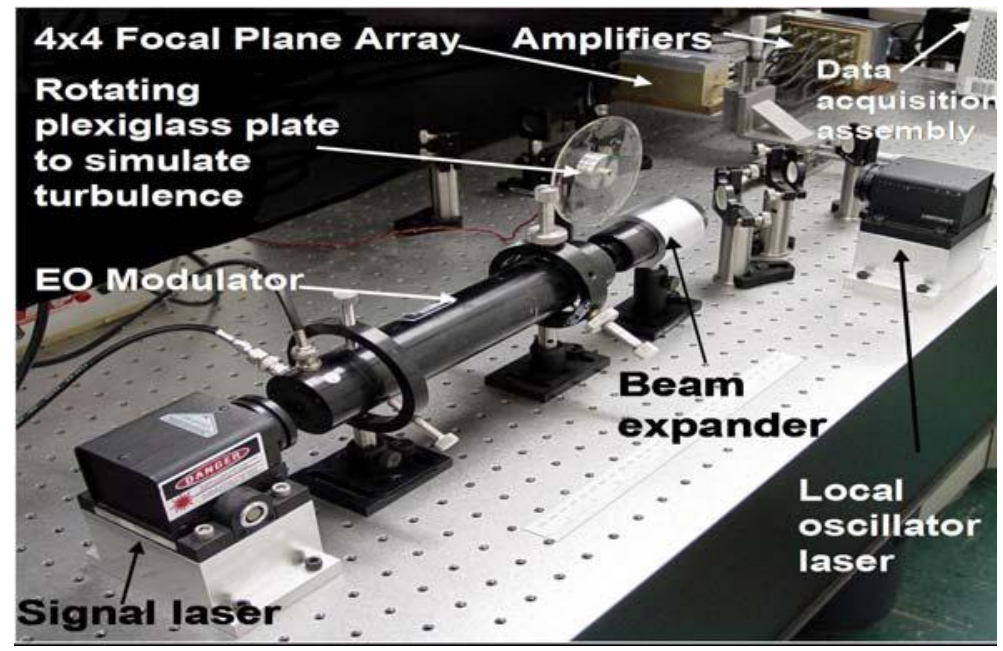

Fig. 4: Coherent combining experiment at the Jet Propulsion Laboratory, NASA. 
In the current coherent combining experiment, each of the 16 outputs of the detector array are amplified, and input to a 16-channel data-acquisition assembly. The analog signals are digitized to 8 bits at a sampling rate of 25 mega-samples per second (MSPS). The data-acquisition system is capable of synchronously recording up to 1 megabyte of data per channel. Five channels that contained significant signal energy were identified, and samples from each channel were collected synchronously. The modulation beatnotes can be observed (Fig. 7) at a rate of approximately $100 \mathrm{kHz}$, resulting in a PPM frame period of approximately $10 \mu \mathrm{s}$, only half of which is used for information with this modulator. Since the slot width is $300 \mathrm{~ns}$, there are approximately 16 disjoint pulse-widths in a half-frame, yielding approximately a 16-PPM communications system with dead-time (this modulator was originally used for Q-switched laser applications, where including a dead-time was appropriate). Note, however, that if the entire frame were used, as would be the case in a realistic communications application, then the entire frame could be used to carry information, resulting in a 32-PPM communications system.

A snapshot of an individual laser pulse that contains the coherently detected PPM beatnote is shown in Fig. 5 .

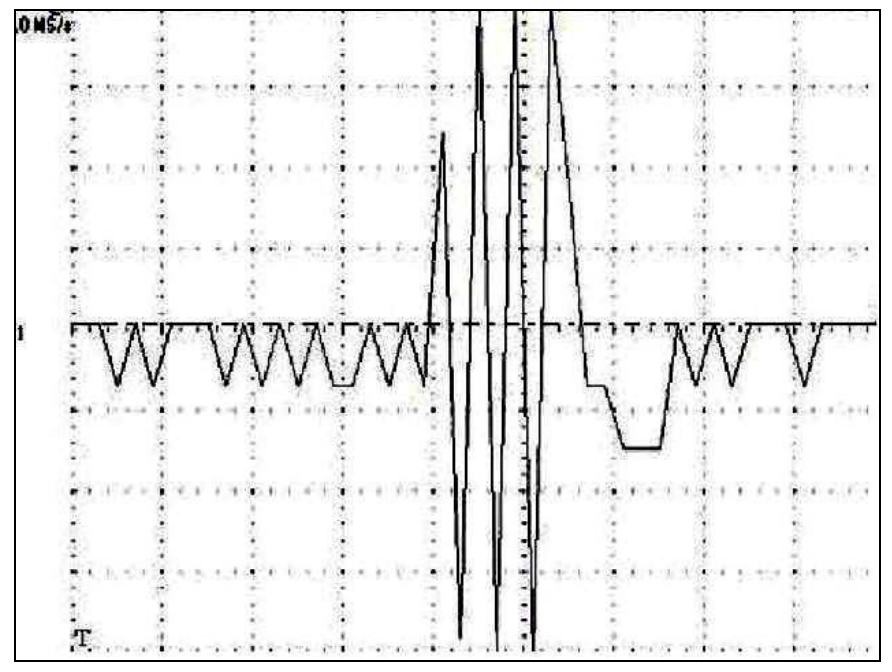

Fig. 5. Snapshot of an individual PPM pulse beatnote.

The optical local oscillator frequency was displaced from the received optical signal frequency by $6 \mathrm{MHz}$, resulting in an intermediate detected frequency of $6 \mathrm{MHz}$. The heterodyned PPM intermediate signal was sampled at $25 \mathrm{MHz}$ ( 40 ns samples), and the resulting sample-stream digitally downconverted to complex baseband (this operation effectively upconverted the $488 \mathrm{KHz}$ laser relaxation oscillation to $6.5 \mathrm{MHz}$, which was subsequently removed from the complex baseband samples by low-pass filtering. The resulting downconverted complex samples served as input to a leastmean-square (LMS) algorithm, which was used to estimate the complex weights required to reconstruct the signal. The complex-weighted samples from each channel were then combined, in order to maximize the combined signal-to-noise ratio (SNR).

A rotating pre-distorted plexiglass plate was incorporated into the experimental setup to simulate atmospheric turbulence. Intensity distributions of the signal beam at the input to the focal-plane array under ideal conditions, and with simulated turbulence respectively are shown from left to right in Fig. 6.

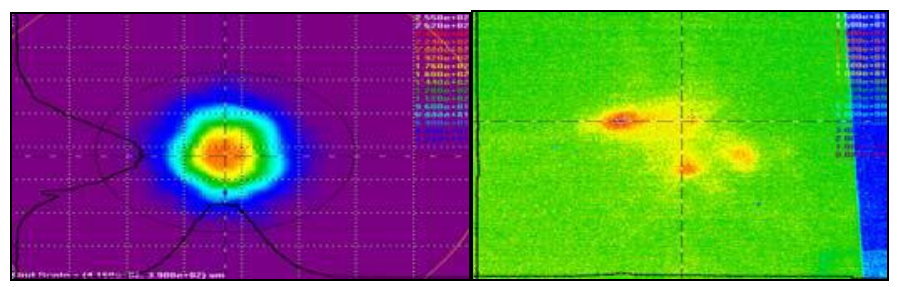

Fig.6: Beam profile under ideal (undistorted) conditions, and with simulated turbulence using a plexiglass plate. 
Fig. 7 illustrates coherently detected PPM beatnotes in five different channels in the presence of atmospheric attenuation.

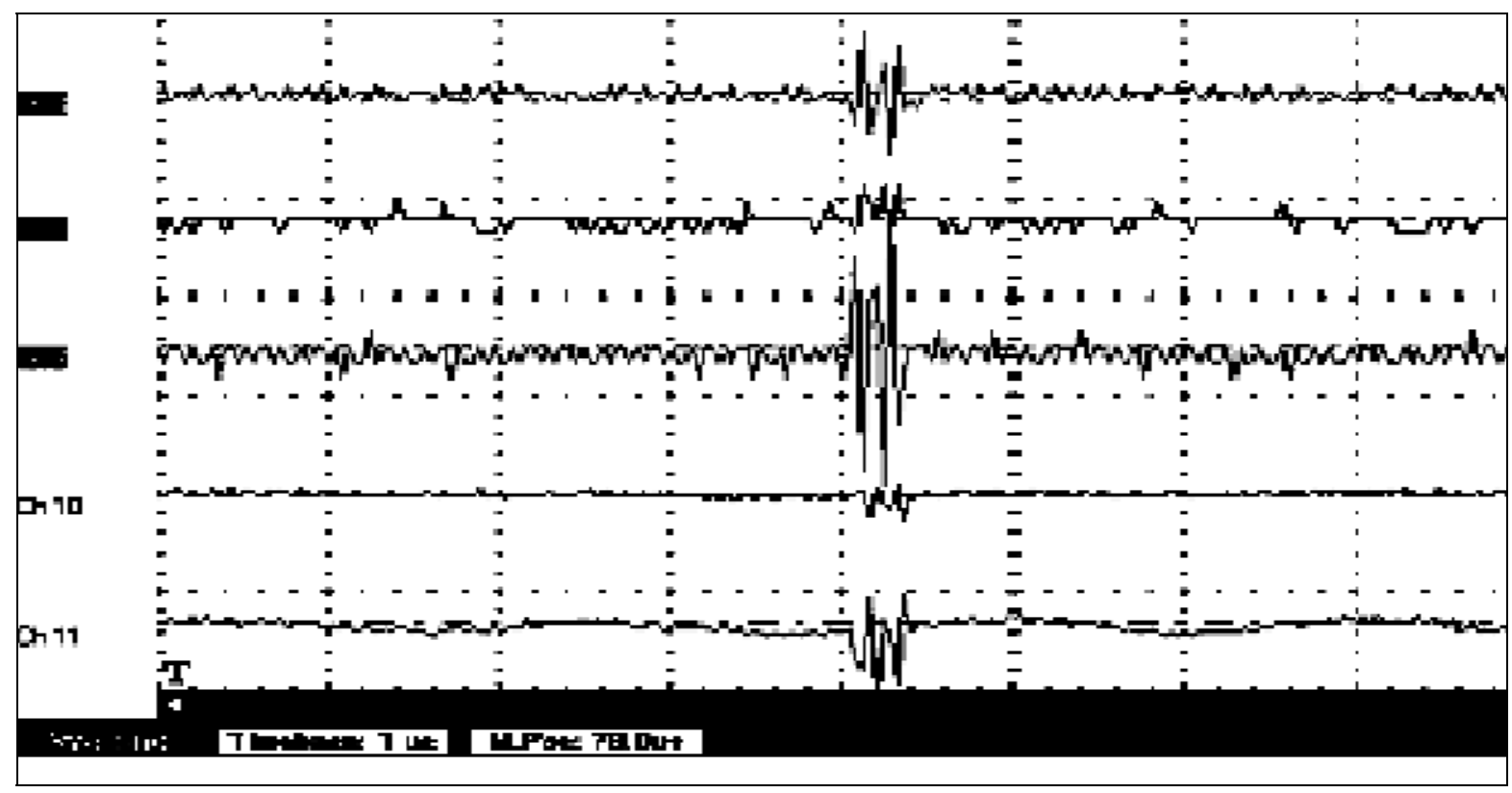

Fig. 7: Sampled sequences of 5 channels containing PPM-modulated 6MHz beat-notes.

\section{ADAPTIVE COMBINING OF BEATNOTES USING THE LMS ALGORITHM}

The discrete complex version of the LMS algorithm can be described by the recursive equation:

$$
W_{i}(n+1)=W_{i}(n)+\mu S_{i}^{*}(n) \varepsilon(n)
$$

The LMS is a recursive algorithm that allows the value of each weight, $W_{i}$, at the $(n+1)$ sample to be calculated from its value at the $\mathrm{n}$-th sample, using the signals at the nth sample. The sampled error signal is obtained from the sampled reference signal and array output, as follows:

$$
\varepsilon(n)=d(n)-s(n)=d(n)-\sum_{i=1}^{N} W_{i}(n) S_{i}(n)
$$

The LMS algorithm described in Eqs. (5) and (6) is complex in the sense that the input and output data as well as the weights are all complex values. In our experiments, the reference signal is a constant value, equal to the sum of the average magnitudes of the signals in the signal channels. The weights are computed from Eq. (5) starting with zero initial values. Varying the stepsize, it is possible to control the fraction of the current weight estimate applied during each update, providing a desired degree of smoothing to the weight estimates.

\subsection{Adaptive combining of simulated data: signal tone and 32-PPM signals}

Convergence of the combining weights as a function of sample number has been analyzed (derivation in Appendix A). Several cases have been considered, including signal tone and PPM signal observed under ideal conditions. Fig. 8 shows the comparison of the convergence of the LMS algorithm for the case of signal tone versus a 32-PPM signal. It has been demonstrated that the number of samples required to obtain convergence in the case of $M$-ary PPM signal 
with a peak power constraint is $M$ times the number of samples required by a continuous tone, due to decreased total signal energy in the lower duty-cycle modulated waveform. Therefore, for the simulated case of a signal tone, convergence is obtained after 4 samples, while for 32-PPM, 128 samples are required for convergence, as illustrated in Fig. 8 for $\mu=1$ and an introduced phase weight variation of 1 radian between every channel.

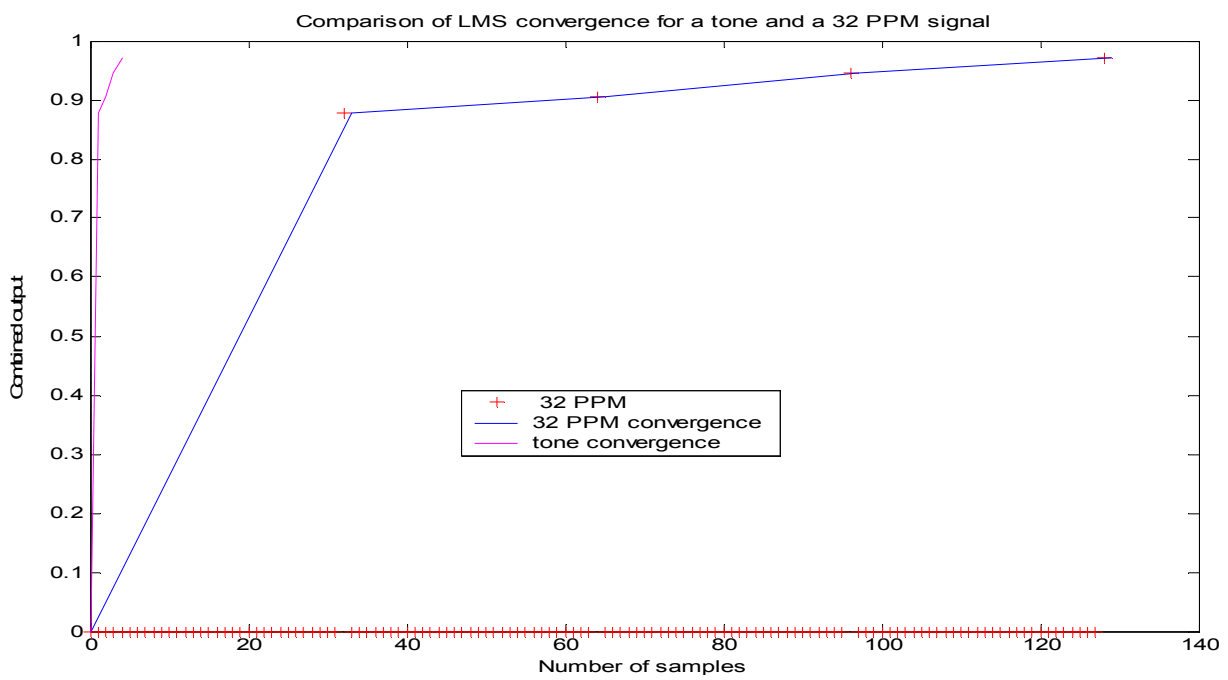

Fig. 8: Comparison of output convergence for signal tone and 32-PPM signal (real part of LMS output).

Fig.9 is a block diagram of the adaptive focal-plane array combining system, where there are $N$ detectors and therefore there are $N$ channels containing signals that undergo amplification, filtering, baseband downconversion and adaptive combining with the LMS algorithm.

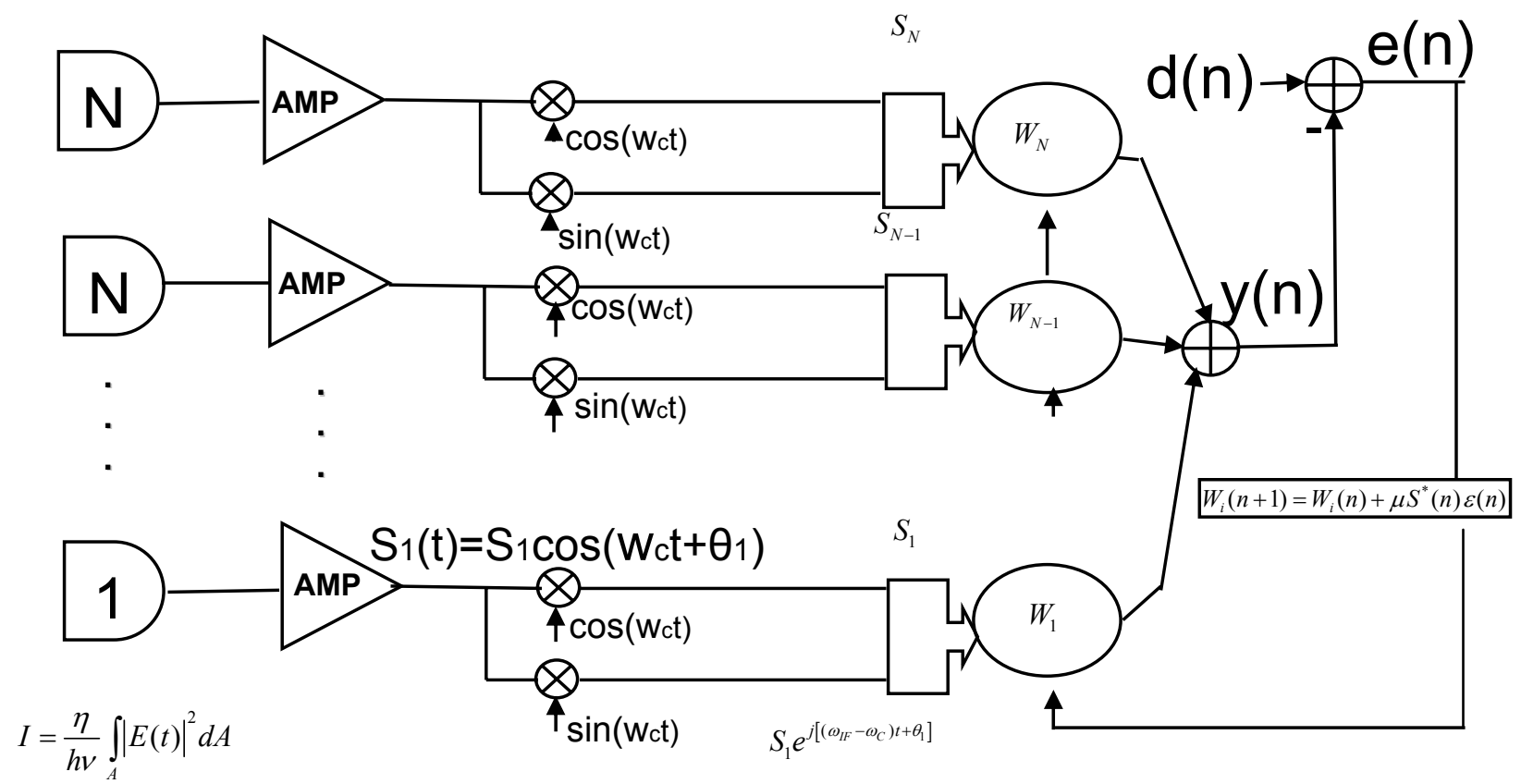

Fig. 9: System block diagram including signal-flow graph representation of the complex LMS algorithm. 
Figure 10 shows a comparison of convergence for a simulated signal tone and a simulated 32-PPM signal where the stepsize is $\mu=0.003$ (detector array consists of 16 detectors). Convergence is obtained for a signal tone after 125 samples, and for the 32-PPM signal after 4000 samples, as expected. Increasing the stepsize to $\mu=0.008$, the LMS algorithm converges faster, hence only 1000 samples are needed for convergence as opposed to 4000 samples for the previous case.

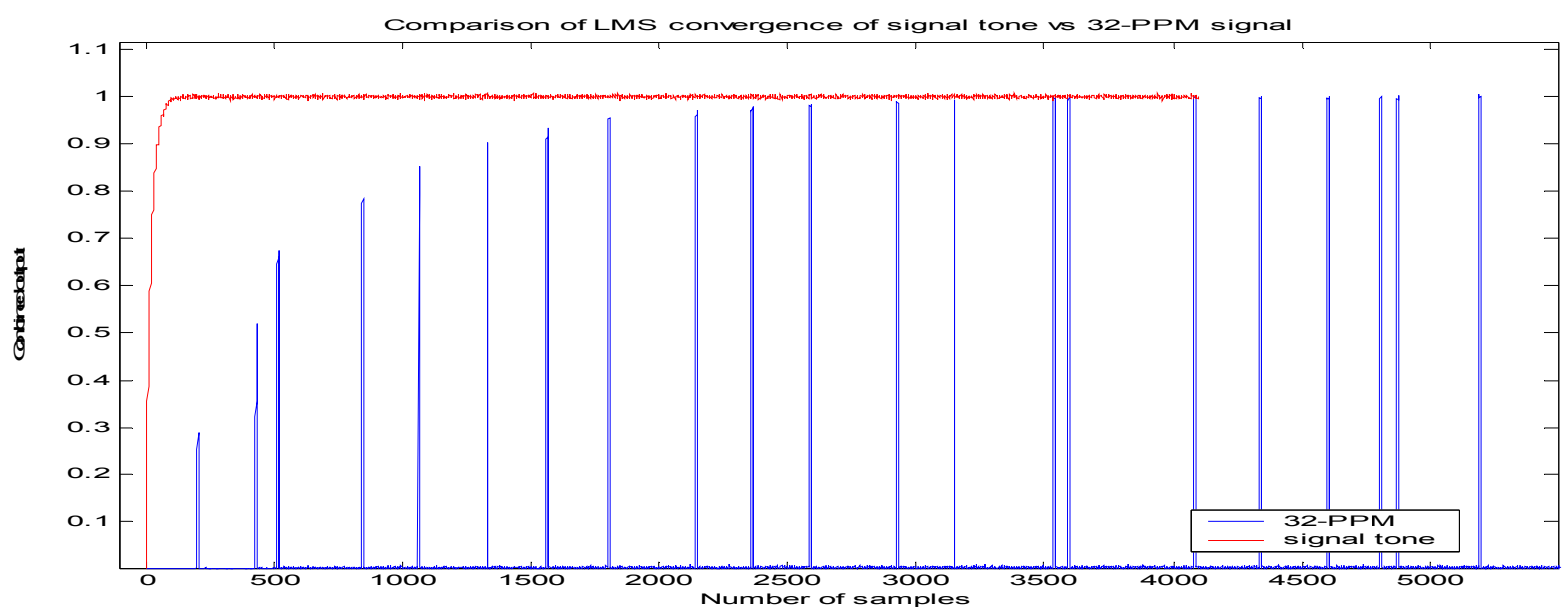

Fig. 10: Comparison of convergence for signal tone and 32-PPM signal

\subsection{Experimental results}

Small stepsize tends to produce accurate weight estimates under static conditions; however the algorithm may not be able to keep up with rapid changes under dynamic conditions with small stepsize. This often leads to "weight misadjustment" errors under dynamic conditions, as the weight estimates cannot keep up with the signal dynamics. Therefore, there is typically a best stepsize to use for each situation.

It is important to note that in the literature, the stepsize is usually taken to be much smaller than one, but that is because the signal is assumed to be of unity amplitude. In our experiment, the signal levels tend to be very small as there is not enough amplification after detection. Therefore, larger values of stepsize are needed to provide adequate updates to the weights.

\subsubsection{Convergence of the LMS algorithm with PPM signals and no atmospheric turbulence}

We first consider the case using a stepsize of $\mu=1$ (considered to be small for the experimentally recorded data, which are on the order of 0.01 for the individual channels). The sum of the magnitudes of the signals in the four selected channels is approximately 0.186 . Fig. 11 shows the combined output (and weighted channel components,) of the LMS combiner for this case; with a stepsize of 1, the LMS algorithm cannot keep up with the phase variations in the beatnote, hence the combined output signal never reaches its maximum value of 0.186 ; instead, it reaches only about 0.037 . 


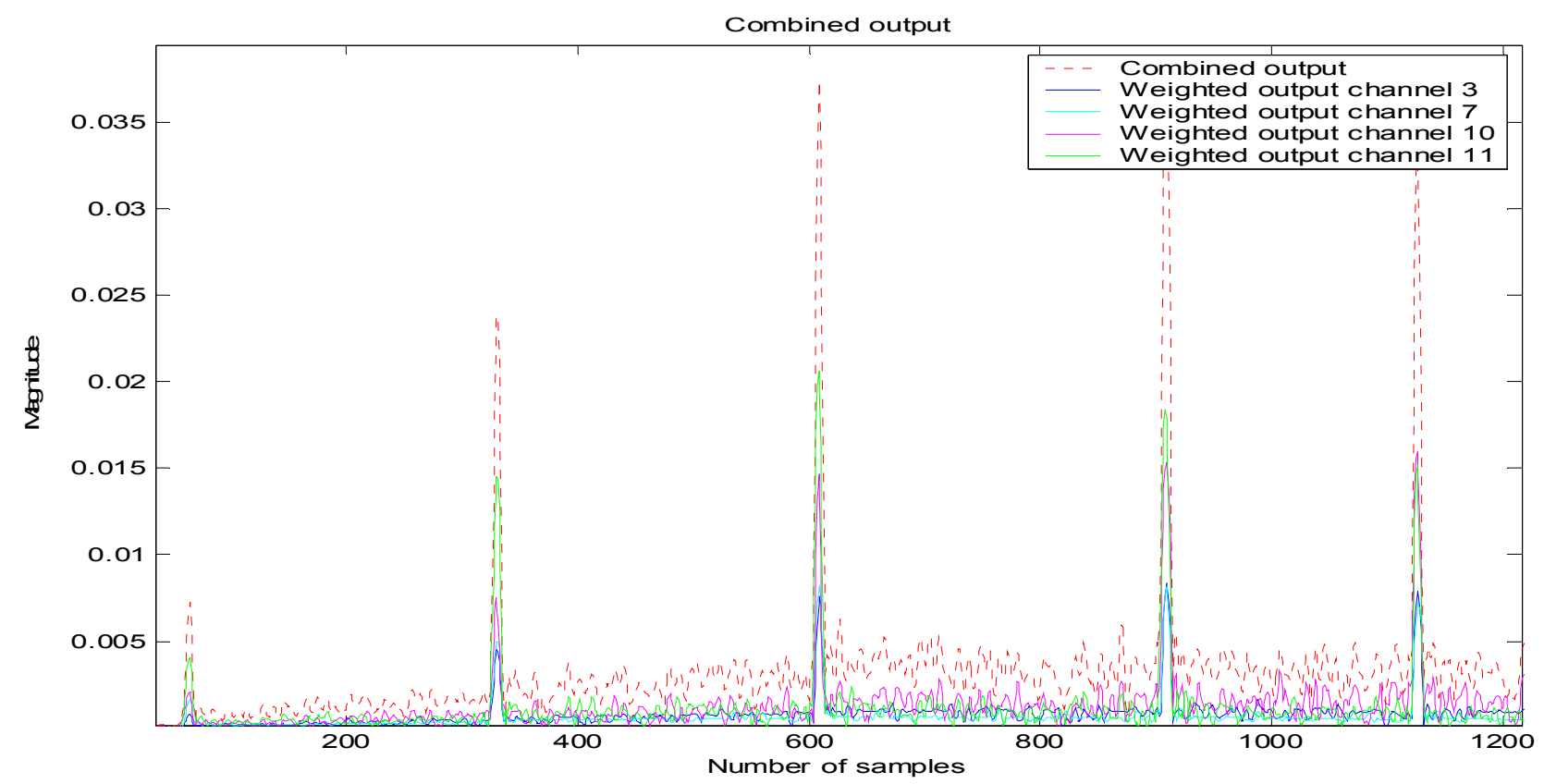

Fig. 11: Combined output with $\mu=1$.

When a larger stepsize is used, $\mu=7$, the combined output achieves the desired value of 0.186 as illustrated in Fig. 12 . We see that the combined output reaches its desired maximum value after approximately 800 samples; this translates to an acquisition time of approximately $32 \mu \mathrm{s}$.

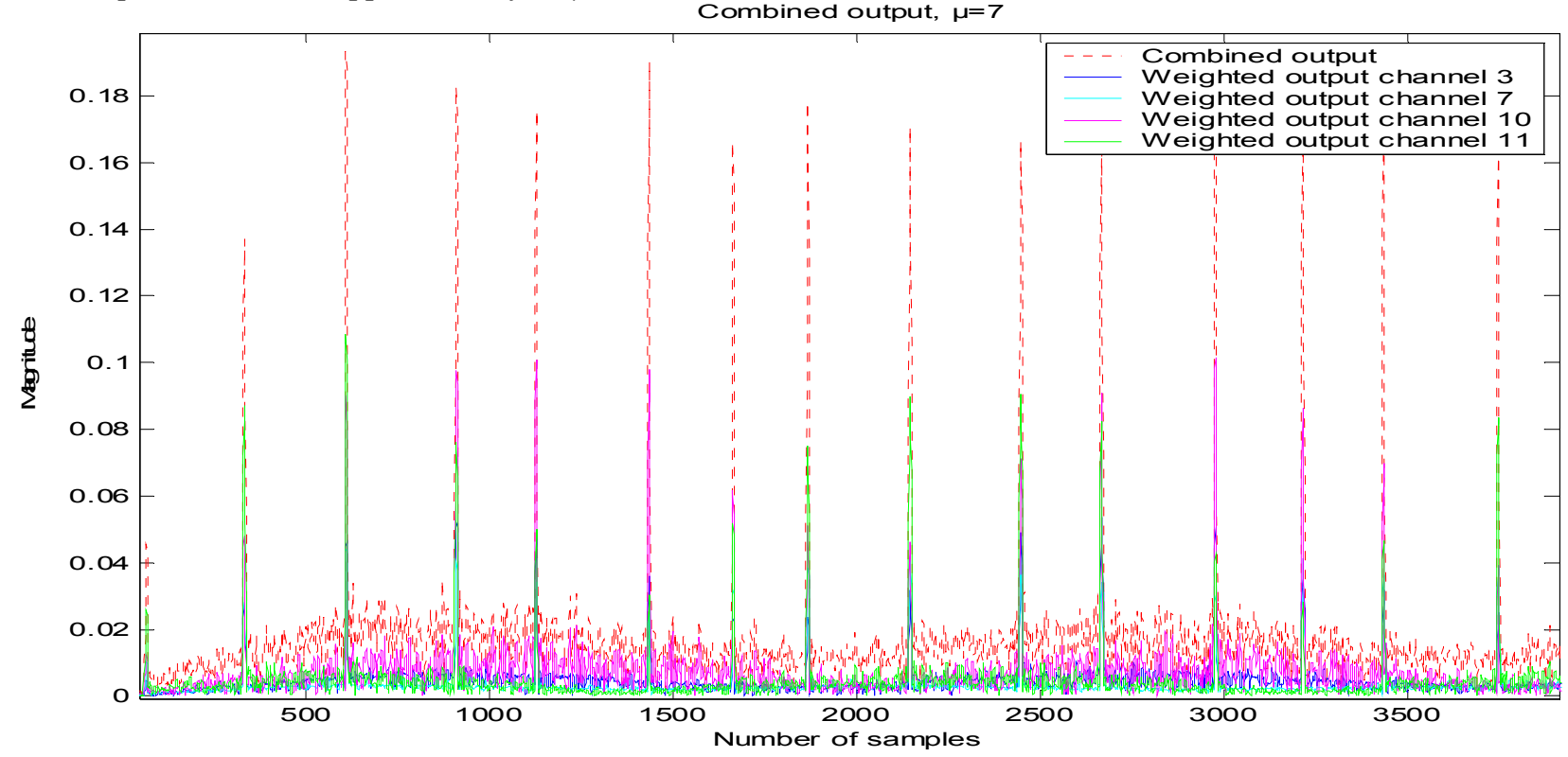

Fig. 12: Combined output with $\mu=7$.

Fig. 13 shows the behavior of the phase of the combining weights, as a function of time (or samples). We observe that the phase of the weights has a sawtooth shape due to the continuously changing phase in the downconverted output, which is not exactly at zero frequency. 


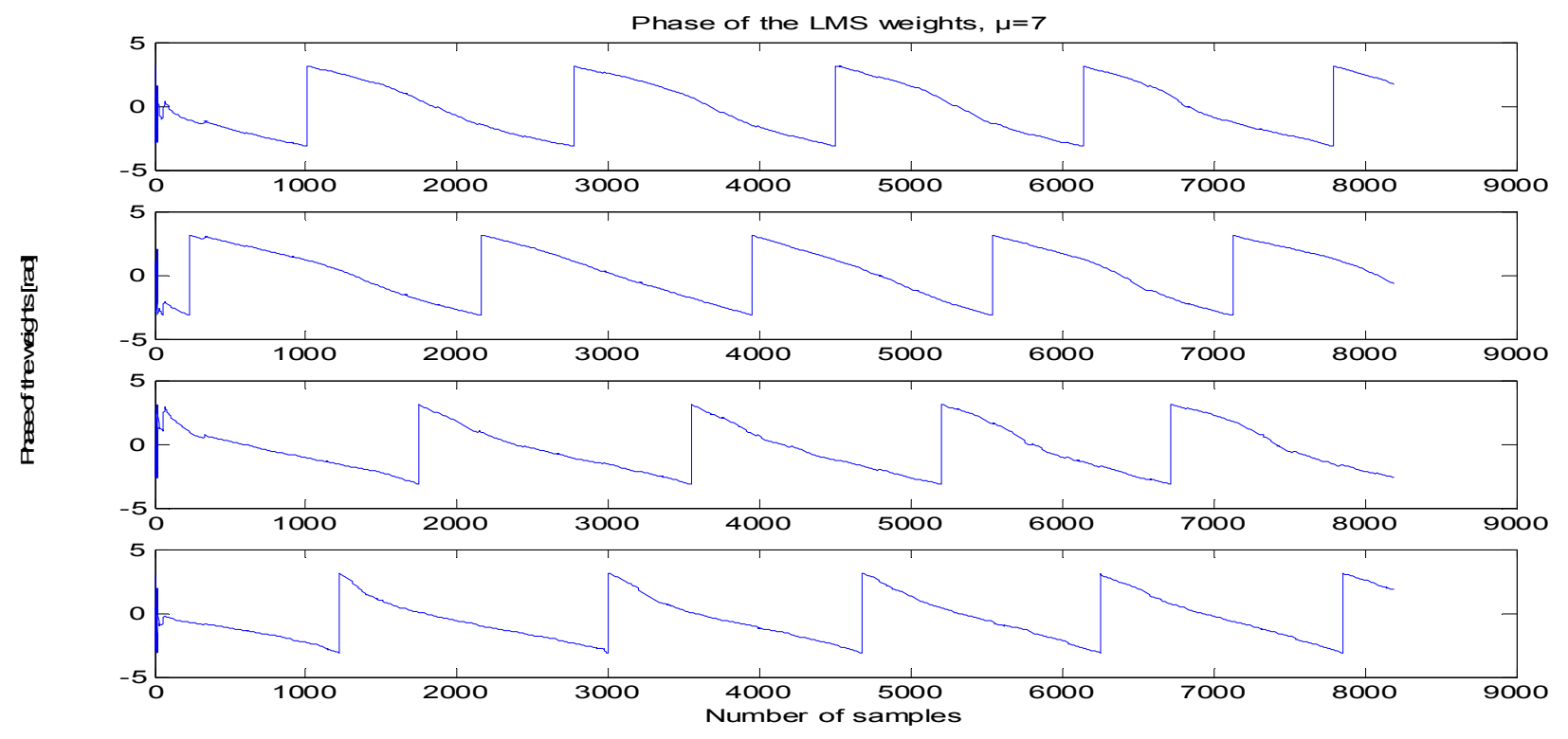

Fig. 13: Phase of the weights for $\mu=7$.

Fig. 14 also shows an individual combined pulse and its weighted components in greater detail. The addition of the magnitudes of the four channels is 0.186 ; indeed, the components sum to the expected value, verifying the validity of the instantaneous combining operation.

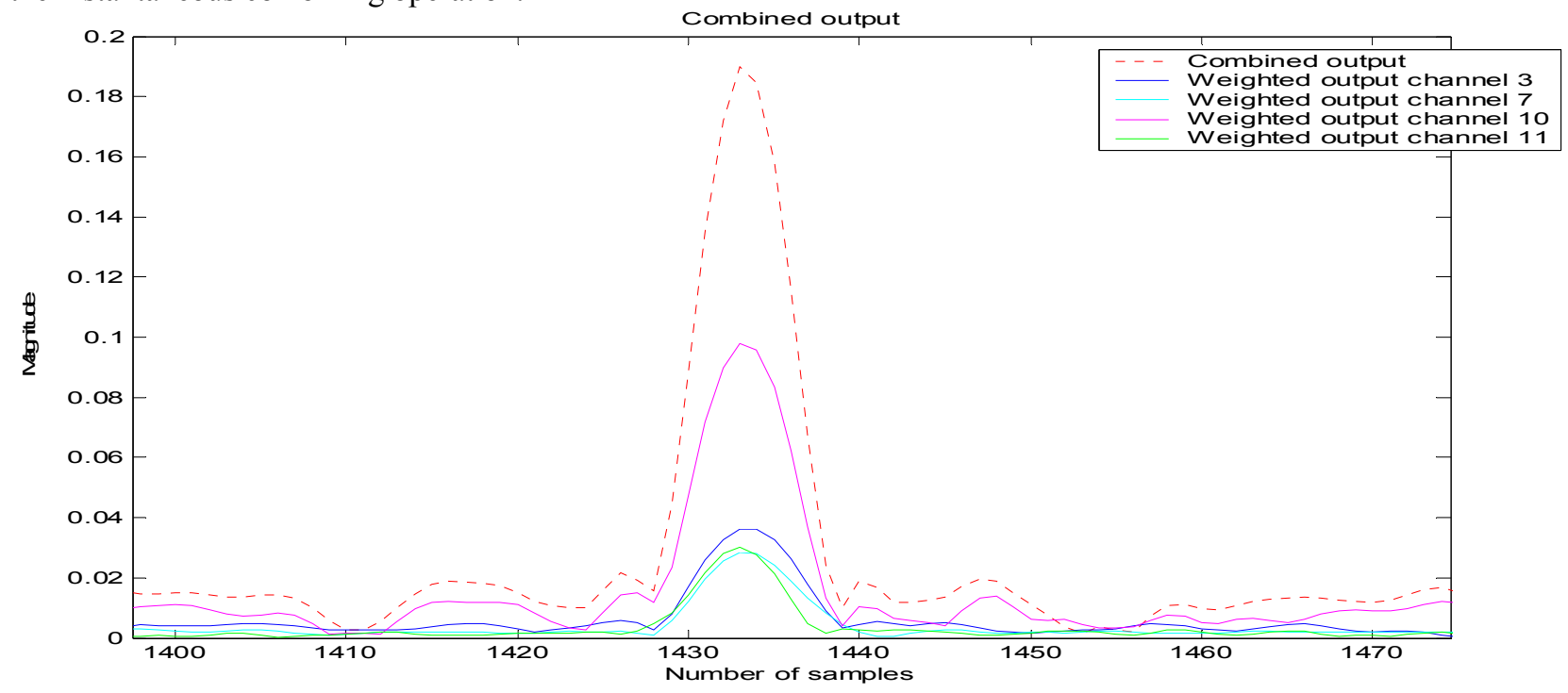

Fig. 14: Combined output and weighted signal components with $\mu=7$.

In summary, it can be seen that the experimentally obtained PPM signals are combined correctly with the larger stepsize, and converge to their desired final value in less than a millisecond.

\subsubsection{Convergence of LMS algorithm in the presence of spatial distortions caused by a static plexiglass plate in the optical path.}

For the case of combining detector array output signals spatially distorted by the plexiglass plate, the desired signal magnitude is the addition of the average magnitudes of the individual channels, which in this case turned out to be 
0.063. Initially, we attempt to combine adaptively using a stepsize of 8, however it can be seen from Fig. 15 that the stepsize is too small hence the LMS algorithm cannot keep up with the residual phase variations and only attains a magnitude of 0.033 .

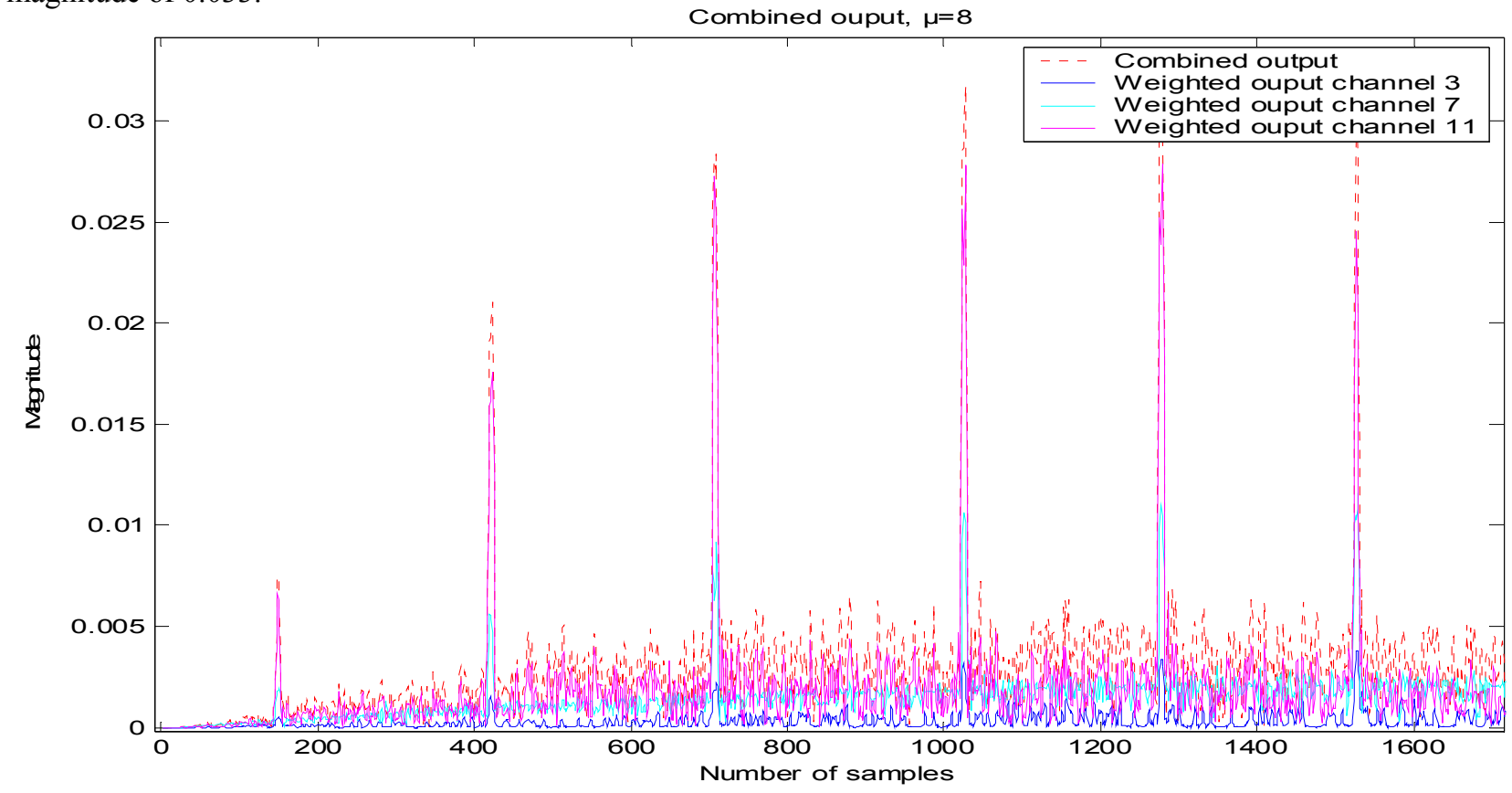

Fig. 15: Combined output with $\mu=8$.

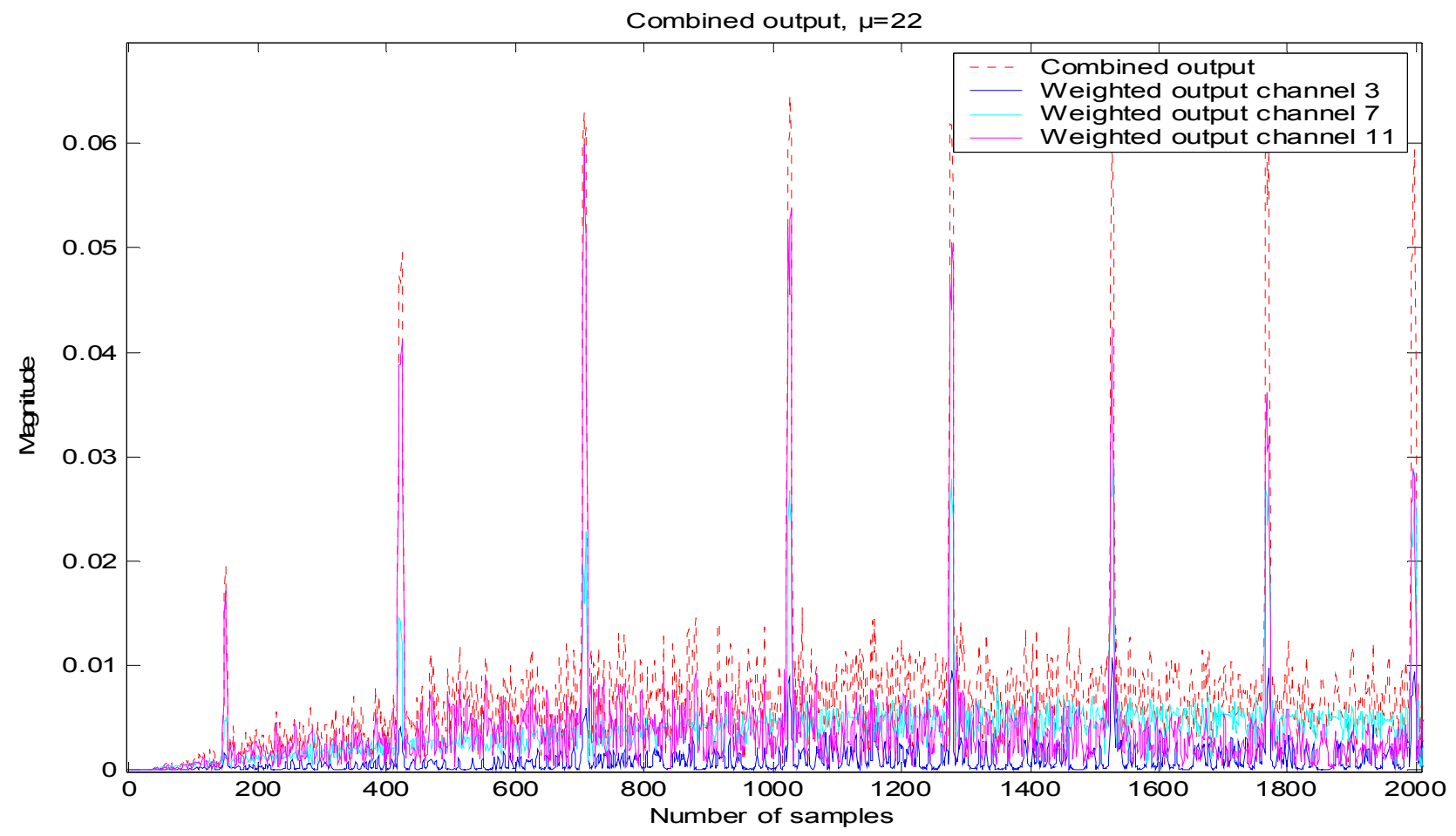

Fig. 16: Combined output with $\mu=20$. 


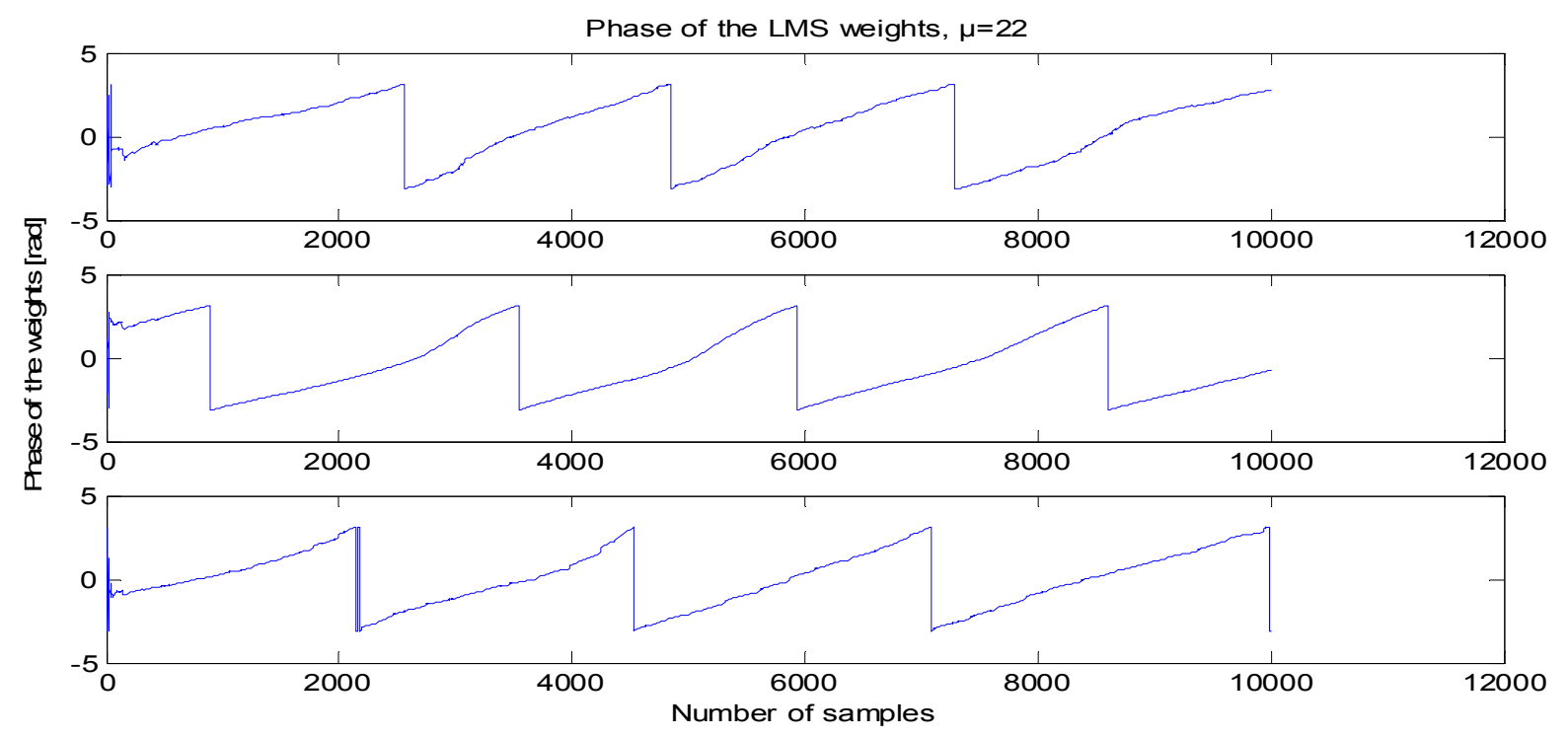

Fig. 17: Phases of the weights for $\mu=22$.

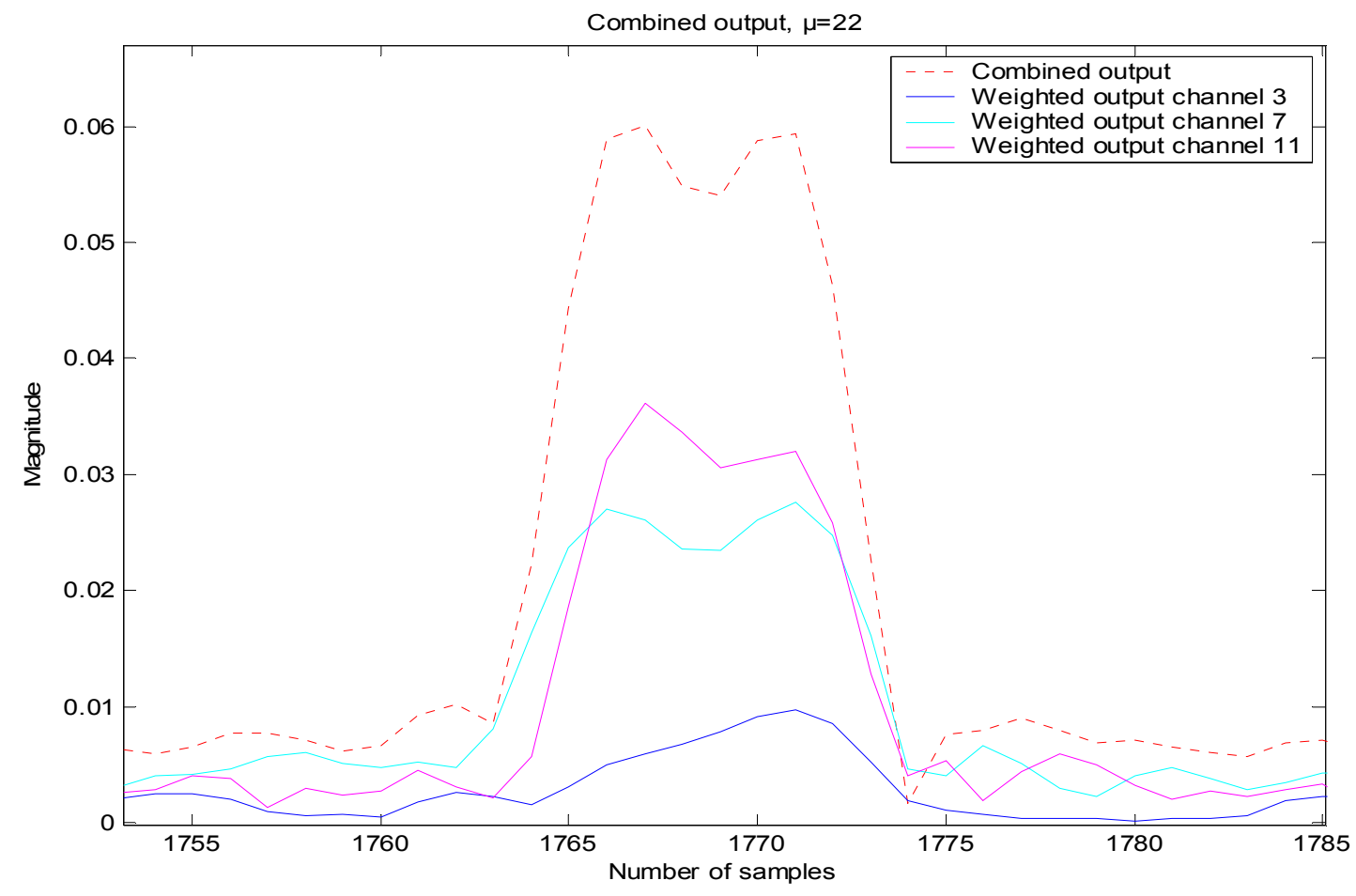

Fig. 18: Combined output with $\mu=22$.

Referring to Figs. 16, 17 and 18, we observe that as we increase the value of the stepsize to $\mu=22$, we get greatly improved combining performance. The combined output shown in Fig. 16 and in more detail in Fig. 18 has increased, approaching its maximum value of 0.063 . At this point, the stepsize is large enough so that the LMS algorithm is able to keep up with the phase-rotation of the complex downconverted beatnote. These results illustrate, that increasing the stepsize allows the LMS algorithm to follow and track the phase-rotation of the complex downconverted beatnote. For 
this value there is accurate tracking of the signals and accordingly, the error signal approaches zero, and maximum combined output is achieved.

\section{CONCLUSIONS AND FUTURE WORK}

We have performed initial testing of an optical coherent communications receiver using PPM signals operating under simulated turbulence conditions. We have shown that a modified LMS algorithm can be used to track the phase of PPM signals generated by the photodetector array, producing an optimally combined signal. Work is continuing to detect the combined PPM signals and verify laboratory performance with theoretical results. Thus far, we have modulated the received optical field with PPM but maintained the pulse-to-pulse coherence of the optical fields enabling the use of a simple modified version of the LMS algorithm. We are also developing new algorithms for the case when the requirements for pulse-to-pulse coherence are relaxed; these algorithms do not depend on temporal coherence on a short time-scale, such as a suitably modified version of a "constant modulus algorithm" (CMA) and other appropriate algorithms for tracking pulsed laser signals received under turbulent conditions.

\section{APPENDIX A}

For a desired signal $d(n)=1, \mu=1$

Assuming that: $\sum\left|S_{i}\right|=1$

$$
\begin{aligned}
& S_{1}=\frac{1}{2}, \quad S_{2}=\frac{1}{2} e^{j \Delta_{2}}, W_{1}(1)=W_{2}(1)=1 \\
& n=1: \\
& \text { 1. } \quad y(1)=\frac{1}{2}+\frac{1}{2} e^{j \Delta_{2}} \\
& \text { 2. } \quad e=\left(1-\frac{1}{2}-\frac{1}{2} e^{j \Delta_{2}}\right)=\frac{1}{2}-\frac{1}{2} e^{j \Delta_{2}} \\
& \text { 3. } \quad e S_{2}^{*}=\left(1-\frac{1}{2}-\frac{1}{2} e^{j \Delta_{2}}\right) \frac{1}{2} e^{-j \Delta_{2}}=\frac{1}{4}\left(e^{-j \Delta_{2}}-1\right) \\
& \quad e S_{1}^{*}=\left(\frac{1}{8}-\frac{1}{8} e^{j \Delta_{2}}\right) \\
& w_{i t h} \quad \mu=1, \\
& W_{2}(2)=1+\frac{1}{4}\left(e^{-j \Delta_{2}}-1\right)=\frac{1}{4}\left(e^{-j \Delta_{2}}+3\right) \\
& W_{1}(2)=\frac{5}{4}-\frac{1}{4} e^{j \Delta_{2}}
\end{aligned}
$$

For the case $n=2$, and $n=3$, the following analysis is shown:

$$
\begin{aligned}
& n=2: \\
& y(2)=\left(\begin{array}{l}
\frac{1}{4}\left(5-e^{j \Delta_{2}}\right) \\
\frac{1}{4}\left(e^{-j \Delta_{2}}+3\right)
\end{array}\right)^{T}\left(\begin{array}{l}
\frac{1}{2} \\
\frac{1}{2} e^{j \Delta_{2}}
\end{array}\right)=\frac{6}{8}+\frac{2}{8} e^{j \Delta_{2}} \\
& e=1-y(2)=\frac{2}{8}-\frac{2}{8} e^{j \Delta_{2}} \\
& e S_{2}^{*}=\left(\frac{2}{8}-\frac{2}{8} e^{j \Delta_{2}}\right) \frac{1}{2} e^{-j \Delta_{2}}=\frac{2}{16} e^{-j \Delta_{2}}-\frac{2}{16} \\
& e S_{1}^{*}=\left(\frac{2}{16}-\frac{2}{16} e^{j \Delta_{2}}\right) \\
& W_{2}(3)=\frac{6}{16} e^{-j \Delta_{2}}+\frac{10}{16} \\
& W_{1}(3)=\frac{22}{16}-\frac{6}{16} e^{j \Delta_{2}}
\end{aligned}
$$

$$
\begin{aligned}
& n=3: \\
& y(3)=\frac{28}{32}+\frac{4}{32} e^{j \Delta_{2}} \\
& e(3)=1-y(3)=\frac{4}{32}-\frac{4}{32} e^{j \Delta_{2}} \\
& e S_{2}^{*}=\frac{4}{64} e^{-j \Delta_{2}}-\frac{4}{64} \\
& e S_{1}^{*}=\left(\frac{4}{64}-\frac{4}{64} e^{j \Delta_{2}}\right) \\
& W_{2}(4)=\frac{36}{64}+\frac{28}{64} e^{-j \Delta_{2}} \\
& W_{1}(4)=\frac{92}{64}-\frac{28}{64} e^{j \Delta_{2}}
\end{aligned}
$$

These weight values were used to compute the output values of the LMS for the example shown in Fig. 10.

\section{ACKNOWLEDGMENTS}

The authors would like to acknowledge Carlos Esproles for his experimental setup contributions.

\section{REFERENCES}

1. Larry C. Andrews, University of Central Florida, Field Guide to Atmospheric Optics, SPIE Field Guides, Volume FG02, John E. Greivenkamp, Series Editor, SPIE Press, Washington 2004.

2. R. M. Gagliardi, S. Karp, Optical Communications, $2^{\text {nd }}$ ed., Wiley Series in Telecommunications and Signal Processing, New York, 1995, Chapter 6.

3. J.C. Palais, Fiber Optic Communications, Prentice Hall, New Jersey, 1998, Chapter 10.

4. M. Muñoz Fernández, V. A. Vilnrotter, Performance analysis and experimental verification of a coherent optical receiver for PPM signals operating in the presence of turbulence, LASE 2004, SPIE Conference, San Jose, CA, U.S.A., January 24-29, 2004. 